\title{
Bending by explosion of a multilayered concrete beam on a visco-elastic basis
}

\author{
Yury Nemirovskii ${ }^{1}$ and Sergey Tikhonov ${ }^{2, *}$ \\ ${ }^{1}$ Khristianovich Institute of Theoretical and Applied Mechanics Siberian Branch of the Russian \\ Academy of Sciences, Physics of Fast Processes Laboratory, 630090 Institutskaya str., 4/1, \\ Novosibirsk, Russia \\ ${ }^{2}$ I. Ulianov Chuvash State University, Faculty of Information and Computer Systems, 428015 \\ Moskovskiy pr., 14, Cheboksary, Russia
}

\begin{abstract}
In this work, the problem of bending of a multilayered concrete beam of arbitrary cross-section by explosive loading on a visco-elastic basis is considered. It is assumed that different grades of concrete can be realized in layers in the cross-section. The property of concrete resistance to tension and compression is considered in work. It is assumed that the dynamic loading is caused by consecutive explosion of two charges over the middle of the span of beam. The distribution of bending moments and deflections of the beam at each time is determined. The time of the end of motion and the residual deflection of beam are found.
\end{abstract}

\section{Introduction}

The beginning of the research of reinforced concrete structures was laid by A. A. Gvozdev [1] who introduced a widely known model of ideal rigid-plastic material into calculation practice and carried out many calculations of the bearing capacity of various building structures on its basis. The model turned out to be quite simple and convenient in design engineering practice and was widely developed [2-6]. It should be noted, however, that even though in the mentioned works it is often noted that the calculations were carried out including the building structures made of reinforced concrete, the specific property of concrete and reinforced concrete structures that is associated with their significant resistance to tension and compression was not taken into account when carrying out specific calculations within the framework of that model [7-9].

In scientific literature, the calculation of reinforced concrete structures is often limited to the case of the simplest forms of rod cross-sections and the simplest conditions of loading and fastening. Modern technological means of creating flexible sets of hybrid laminated structures, where in cross-section different grades of concrete can be realized in layers, are not considered.

In construction practice, there are often situations when deformation is accompanied by a repulse of environment [10] and there is a problem of estimating the bearing capacity of a structure and reducing its damage level in the presence of such repulse under the influence

\footnotetext{
* Corresponding author: strangcheb@mail.ru
} 
of dynamic loads. A similar problem of dynamic loading of a multilayered pinched beam on a viscoelastic basis was considered in [11-12].

Materials included in the real construction in the field of plastic deformations can have completely different dependences. An analysis of possible general relations for the description of the stress-strain state of structures made of hybrid reinforced concrete is given in [13].

\section{Methods}

Creating of a calculation method with a search of all known dependencies is completely unreal and impractical. Therefore, we previously introduce and use further some useful concepts to develop a uniform method of calculation. First, plastically equivalent materials [14].

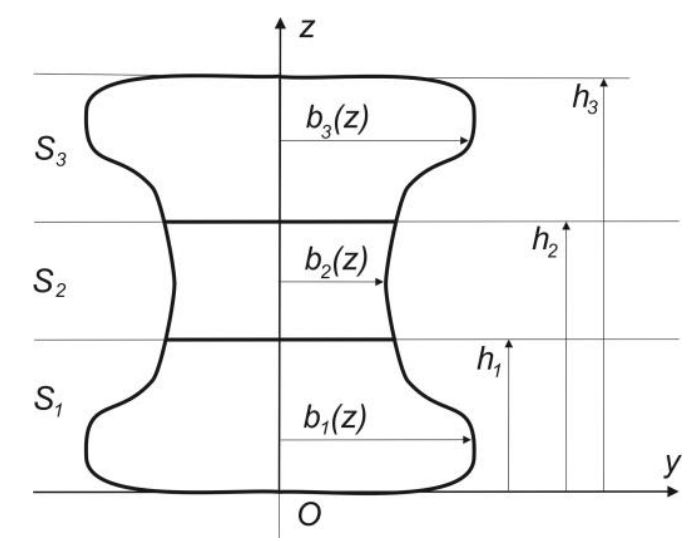

Fig. 1. Cross-section of a three-layered concrete beam

Suppose that considered as a part of a layered beam (fig. 1) $i$-material in plastic area of deformation is described by the dependence

$$
\sigma_{i}=A_{1 i} \varepsilon+A_{2 i} \varepsilon^{2},-\varepsilon_{*_{i}}^{-} \leq \varepsilon \leq \varepsilon_{*_{i}}^{+}
$$

where $\varepsilon_{*_{i}}^{-}, \varepsilon_{*_{i}}^{+}$are maximum allowable strains at compression and tension respectively, $A_{1 i}, A_{2 i}$ are experimentally determined coefficients.

Assume that the coefficients $A_{1 i}, A_{2 i}$ are determined from experiments performed for samples of the corresponding phase materials, for example, by the method of least squares. For this purpose, it is necessary to have real diagrams of tension-compression. As the published data of specific tests are usually not sufficiently complete, the following simplified method of calculation can be used to determine the parameters.

We assume that the modulus of concrete elasticity $E_{i}$ under compression and tension is identical. We assume that concrete behaves like an elastic body in the segment $0 \leq \varepsilon \leq \varepsilon_{*_{i}}^{+}$, to determine $\varepsilon_{*_{i}}^{+}$, from where we can obtain

$$
\varepsilon_{*_{i}}^{+}=\frac{\sigma_{*_{i}}^{+}}{E_{i}},
$$

where $\sigma_{*_{i}}^{+}$is a limit of tensile strength.

From the ratio (1), we can obtain 


$$
\frac{d \sigma_{i}}{d \varepsilon}=A_{1 i}+2 A_{2 i} \varepsilon, \frac{d^{2} \sigma_{i}}{d \varepsilon^{2}}=2 A_{2 i}
$$

From ratios (3), considering that approximating curve described by equation (1) has a convexity down, we have $A_{2 i}>0$. Passing to the limit in the first equation of ratios (3), we get

$$
\lim _{\varepsilon \rightarrow 0} \frac{d \sigma_{i}}{d \varepsilon}=A_{1 i}
$$

where it follows that

$$
A_{1 i}=E_{i},
$$

where $E_{i}$ is a modulus of concrete elasticity.

Considering that the point $\left(-\sigma_{*_{i}}^{-},-\varepsilon_{*_{i}}^{-}\right)$in the equation (1) must be an extremum point where $\sigma_{*_{i}}^{-}$is a limit strength at compression, we obtain

$$
\sigma_{*_{i}}^{-}=A_{1 i} \varepsilon_{*_{i}}^{-}-A_{2 i}\left(\varepsilon_{*_{i}}^{-}\right)^{2}, \frac{d \sigma_{i}}{d \varepsilon}=E_{i}-2 A_{2 i} \varepsilon_{*_{i}}^{-}=0
$$

therefore

$$
\varepsilon_{*_{i}}^{-}=2 \frac{\sigma_{*_{i}}^{-}}{E_{i}}, A_{2 i}=\frac{1}{4} \frac{\left(E_{i}\right)^{2}}{\sigma_{*_{i}}^{-}} .
$$

Thus, it is sufficient to have three traditional characteristics $E_{i}, \sigma_{*_{i}}^{-}, \sigma_{*_{i}}^{+}$when using the approximation (1).

We consider ideally plastic materials with yield limits respectively $\sigma_{0 i}^{+}, \sigma_{0 i}^{-}$on the deformation segments $0 \leq \varepsilon \leq \varepsilon_{*_{i}}^{+},-\varepsilon_{*_{i}}^{-} \leq \varepsilon \leq 0$ on the deformation segments. along with approximating ratio (1). Consider these materials plastically equivalent [12] if they perform the identical work on deformation

$$
\int_{0}^{\varepsilon_{*_{i}}^{+}} \sigma_{0 i}^{+} d \varepsilon=\int_{0}^{\varepsilon_{*_{i}}^{+}}\left(A_{1 i} \varepsilon+A_{2 i} \varepsilon^{2}\right) d \varepsilon, \int_{-\varepsilon_{i_{i}}^{-}}^{0} \sigma_{0 i}^{-} d \varepsilon=-\int_{-\varepsilon_{i_{i}}^{-}}^{0}\left(A_{1 i} \varepsilon+A_{2 i} \varepsilon^{2}\right) d \varepsilon
$$

where we get

$$
\sigma_{0 i}^{+}=\frac{A_{2 i}\left(\varepsilon_{*_{i}}^{+}\right)^{2}}{3}+\frac{A_{1 i} \varepsilon_{*_{i}}^{+}}{2}, \sigma_{0 i}^{-}=\frac{A_{1 i} \varepsilon_{*_{i}}^{-}}{2}-\frac{A_{2 i}\left(\varepsilon_{*_{i}}^{-}\right)^{2}}{3} .
$$

Substituting in expressions (7) the values of coefficients obtained in the ratios (2), (4), (6), we get

$$
\sigma_{0 i}^{-}=\frac{2}{3} \sigma_{*_{i}}^{-}, \sigma_{0 i}^{+}=\frac{\left(\sigma_{*_{i}}^{+}\right)^{2}}{12 \sigma_{*_{i}}^{-}}+\frac{\sigma_{*_{i}}^{+}}{2}, \varepsilon_{*_{i}}^{-}=2 \frac{\sigma_{*_{i}}^{-}}{E_{i}}, \varepsilon_{*_{i}}^{+}=\frac{\sigma_{*_{i}}^{+}}{E_{i}} .
$$

Substitute all layers $S_{i}$ in fig. 1 for plastically equivalent ideal materials. Then the expression for the force $N$ and the bending moment $M$ has the form

$$
\begin{gathered}
N=2 \int_{0}^{h_{1}} \sigma_{01}^{+} b_{1}(z) d z+2 \int_{h_{1}}^{z_{1}} \sigma_{02}^{+} b_{2}(z) d z-2 \int_{z_{1}}^{h_{2}} \sigma_{02}^{-} b_{2}(z) d z-2 \int_{h_{2}}^{h_{3}} \sigma_{03}^{-} b_{3}(z) d z= \\
=F_{1}-\left(\sigma_{02}^{+}+\sigma_{02}^{-}\right) \varphi_{2}\left(z_{1}\right), \\
M=2 \int_{0}^{h_{1}} \sigma_{01}^{+} b_{1}(z) z d z+2 \int_{h_{1}}^{z_{1}} \sigma_{02}^{+} b_{2}(z) z d z-2 \int_{z_{1}}^{h_{2}} \sigma_{02}^{-} b_{2}(z) z d z-2 \int_{h_{2}}^{h_{3}} \sigma_{03}^{-} b_{3}(z) z d z=
\end{gathered}
$$




$$
=F_{2}-\left(\sigma_{02}^{+}+\sigma_{02}^{-}\right) \psi_{2}\left(z_{1}\right)
$$

where

$$
\begin{gathered}
F_{1}=\sigma_{01}^{+}\left(\varphi_{1}\left(h_{1}\right)-\varphi_{1}(0)\right)-\left(\sigma_{02}^{+} \varphi_{2}\left(h_{1}\right)+\sigma_{02}^{-} \varphi_{2}\left(h_{2}\right)\right)-\sigma_{03}^{-}\left(\varphi_{3}\left(h_{3}\right)-\varphi_{3}\left(h_{2}\right)\right), \\
F_{2}=\sigma_{01}^{+}\left(\psi_{1}\left(h_{1}\right)-\psi_{1}(0)\right)-\left(\sigma_{02}^{+} \psi_{2}\left(h_{1}\right)+\sigma_{02}^{-} \psi_{2}\left(h_{2}\right)\right)-\sigma_{03}^{-}\left(\psi_{3}\left(h_{3}\right)-\psi_{3}\left(h_{2}\right)\right), \\
\varphi_{i}(z)=2 \int b_{i}(z) d z, \quad \psi_{i}(z)=2 \int b_{i}(z) z d z, \quad(i=1,2,3) .
\end{gathered}
$$

According to Kirchhoff-Lyav's hypothesis, we have in examined case

$$
h_{1} \leq z_{1}=-\frac{\varepsilon_{0}}{\kappa} \leq h_{2}, \quad \varepsilon_{0}>0, \kappa<0 .
$$

Assuming $N=0$ in equation (9), we obtain an expression for $z_{1}$

$$
z_{1}=\varphi_{2}^{-1}\left(\frac{F_{1}}{\sigma_{02}^{+}+\sigma_{02}^{-}}\right),
$$

where $\varphi_{2}^{-1}$ is an inverse function to $\varphi_{2}$.

Substituting the value found in (4), we obtain the value of limit bending moment

$$
M_{0}^{+}=F_{2}-\left(\sigma_{02}^{+}+\sigma_{02}^{-}\right) \psi_{2}\left(\varphi_{2}^{-1}\left(\frac{F_{1}}{\sigma_{02}^{+}+\sigma_{02}^{-}}\right)\right) \text {. }
$$

\section{Results}

Consider the case when the beam is hinged. Two states rigid $0 \leq x \leq x_{1}(t)$ and plastic $x_{1}(t) \leq x \leq \frac{a}{2}$ can be realized in the beam (fig. 2).

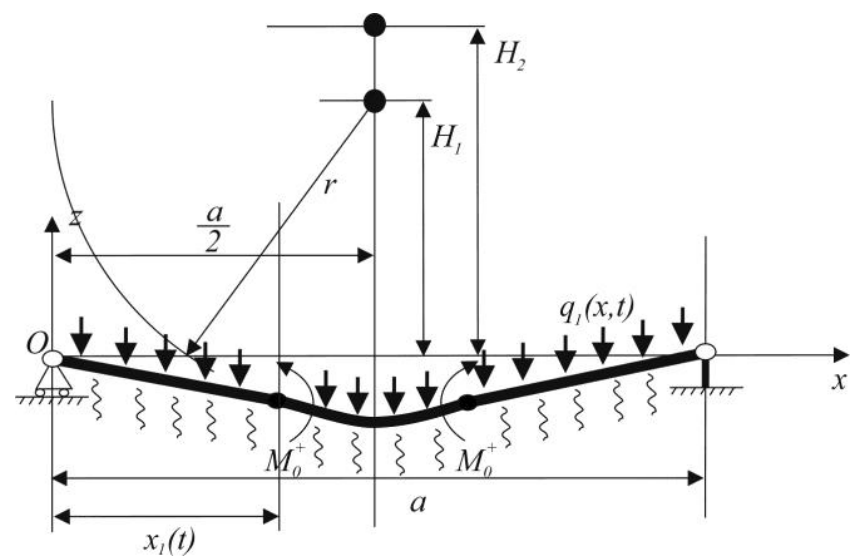

Fig. 2. The scheme of beam deformation

Consider that the load at explosion of the first charge can be represented in the form

$$
q_{i}(x, t)=f_{0 i}(x) e^{-\alpha_{i} t},
$$

where $f_{0 i}(x)=q_{0 i} p_{i}(x), q_{0 i}, \alpha_{i}$ are charge parameters, $p_{1}(x)$ is a distribution function of the blast wave, depending on the location of charge, which we take in the form [15] 


$$
f_{0 i}(x)=q_{0 i} b_{0 i}\left(\frac{r}{R_{0 i}}\right)^{-a_{i}-1}=q_{0 i} b_{0 i} R_{0 i}^{a_{i}+1}\left(H_{i}^{2}+\left(\frac{a}{2}-x\right)^{2}\right)^{\frac{-a_{i}-1}{2}},
$$

where $b_{01}, R_{01}, a_{1}$ are parameters determined from the experiment.

Then the equation of dynamic bending has the form

$$
\frac{\partial^{2} M}{\partial x^{2}}+q_{1}(x, t)+q_{s}-m \frac{\partial^{2} w}{\partial t^{2}}-k_{1} \frac{\partial w}{\partial t}-k_{2} w=0
$$

where $m$ is a distributed mass of the beam, $w$ is a deflection, $q_{s}$ is a distributed weight of the beam, $k_{1}, k_{2}$ are coefficients of viscous and elastic resistance of the basis.

For rigid segment the curvature $\kappa(x)=-\frac{\partial^{2} w(x, t)}{\partial x^{2}}=0$, then from the condition of fixing the left end of the beam

$$
w(0, t)=0, M(0, t)=0,
$$

and condition at the border of two areas

$$
Q\left(x_{1}(t), t\right)=0,
$$

get expressions for the deflection and the moment in section $0 \leq x \leq x_{1}(t)$

$$
\begin{gathered}
w(x, t)=\theta_{1}(t) x \\
M(x, t)=-\int_{0}^{x}\left(\int_{x_{1}(t)}^{x}\left(q_{1}(x, t)+q_{s}\right) d x\right) d x+\left(m \frac{\partial^{2} \theta_{1}(t)}{\partial t^{2}}+k_{1} \frac{\partial \theta_{1}(t)}{\partial t}+k_{2} \theta_{1}(t)\right)\left(\frac{x^{3}}{6}-\frac{x_{1}^{2}(t)}{2} x\right),
\end{gathered}
$$

and $M(x, t)<M_{0}^{+}$should be fair everywhere the site $0 \leq x<x_{1}(t)$.

In plastic area, the moment has reached its limit value

$$
M(x, t)=M_{0}^{+}, \quad x_{1} \leq x \leq \frac{a}{2},
$$

then for the specified section we obtain the differential equation for determining the deflection in plastic region

$$
\frac{\partial^{2} w}{\partial t^{2}}+\frac{k_{1}}{m} \frac{\partial w}{\partial t}+\frac{k_{2}}{m} w=f_{01}(x) e^{-\alpha_{1} t}+f_{1},
$$

where

$$
f_{1}=\frac{q_{s}}{m} .
$$

Equation (20) is a second-order linear inhomogeneous differential equation with constant coefficients.

Initial conditions of the equation (20) is

$$
\left.w(x, t)\right|_{t=0}=\left.\frac{\partial w(x, t)}{\partial t}\right|_{t=0}=0 .
$$

Depending on the discriminant of characteristic equation (19) $d=\frac{k_{1}^{2}}{m^{2}}-4 \frac{k_{2}}{m}$, three cases are possible when solving the equation (19).

Consider the first case, which takes place if $d>0$. The remaining two cases are considered similarly.

Then the general solution of equation (20) has the form

$$
w(x, t)=C_{1}(x) e^{f_{2} t}+C_{2}(x) e^{f_{3} t}+\frac{f_{01}(x) e^{-\alpha_{1} t}}{\left(f_{2}+\alpha_{1}\right)\left(f_{3}+\alpha_{1}\right)}+\frac{f_{1}}{f_{2} f_{3}},
$$


for the deflection speed it is true

$$
\frac{\partial w(x, t)}{\partial t}=f_{2} C_{1}(x) e^{f_{2} t}+f_{3} C_{2}(x) e^{f_{3} t}-\frac{f_{01}(x) \alpha_{1} e^{-\alpha_{1} t}}{\left(f_{2}+\alpha_{1}\right)\left(f_{3}+\alpha_{1}\right)} .
$$

The solution with the boundary condition (20) has the form

$$
w(x, t)=\left(f_{4} f_{01}(x)+f_{5}\right) e^{f_{2} t}+\left(f_{6} f_{01}(x)+f_{7}\right) e^{f_{3} t}+f_{8} f_{01}(x) e^{-\alpha_{1} t}+f_{9},
$$

where

$$
\begin{aligned}
f_{4}=\frac{1}{\left(f_{2}+\alpha_{1}\right)\left(f_{2}-f_{3}\right)}, f_{5} & =\frac{f_{1}}{f_{2}\left(f_{2}-f_{3}\right)}, f_{6}=\frac{1}{\left(f_{3}+\alpha_{1}\right)\left(f_{3}-f_{2}\right)}, f_{7}=\frac{f_{1}}{f_{3}\left(f_{2}-f_{3}\right)}, \\
f_{8} & =\frac{1}{\left(f_{2}+\alpha_{1}\right)\left(f_{3}+\alpha_{1}\right)}, f_{9}=\frac{f_{1}}{f_{2} f_{3}} .
\end{aligned}
$$

We rewrite equation (24) as

$$
w(x, t)=f_{01}(x)\left(f_{4} e^{f_{2} t}+f_{6} e^{f_{3} t}+f_{8} e^{-\alpha_{1} t}\right)+f_{5} e^{f_{2} t}+f_{7} e^{f_{3} t}+f_{9} .
$$

From (25) we can obtain the expression for the speed of deflection

$$
\frac{\partial w(x, t)}{\partial t}=f_{01}(x)\left(f_{4} f_{2} e^{f_{2} t}+f_{6} f_{3} e^{f_{3} t}-\alpha_{1} f_{8} e^{-\alpha_{1} t}\right)+f_{5} f_{2} e^{f_{2} t}+f_{7} f_{3} e^{f_{3} t} .
$$

At the boundary of rigid and plastic states, the conditions of deflection continuity should be true

$$
\left.[w(x, t)]\right|_{x=x_{1}(t)}=0,
$$

where the symbol $[\ldots]$ denotes the jump of the considered quantity.

From (17), (25), (27) we obtain the expression for the deflection for a rigid section

$$
w(x, t)=\left(f_{01}\left(x_{1}(t)\right)\left(f_{4} e^{f_{2} t}+f_{6} e^{f_{3} t}+f_{8} e^{-\alpha_{1} t}\right)+f_{5} e^{f_{2} t}+f_{7} e^{f_{3} t}+f_{9}\right) \frac{x}{x_{1}(t)} .
$$
form

The continuity condition of the deflection speed at the boundary of two regions has the

$$
\left.\left[\frac{\partial w(x, t)}{\partial x}\right]\right|_{x=x_{1}(t)}=0
$$

From (25), (28), (29) you can obtain an equation to determine the boundary $x_{1}(t)$

$$
\left.x_{1}(t) f_{01}^{\prime}(x)\right|_{x=x_{1}(t)}-f_{01}\left(x_{1}(t)\right)=\frac{f_{5} e^{f_{2} t}+f_{7} e^{f_{3} t}+f_{9}}{f_{4} e^{f_{2} t}+f_{6} e^{f_{3} t}+f_{8} e^{-\alpha_{1} t}},
$$

where the function $f_{01}(x)$ has the form (13).

The first limit load can be found from the expression $x_{1}(t)$ found in (30) by putting $x_{1}(0)=\frac{a}{2}$.

From equation (26) we can find the time of stop of the structure motion $t_{f}$

$$
f_{01}\left(x_{1}\left(t_{f}\right)\right)\left(f_{4} f_{2} e^{f_{2} t_{f}}+f_{6} f_{3} e^{f_{3} t_{f}}-\alpha_{1} f_{8} e^{-\alpha_{1} t_{f}}\right)+f_{5} f_{2} e^{f_{2} t_{f}}+f_{7} f_{3} e^{f_{3} t_{f}}=0 .
$$

The residual deflection is determined from equation (28) at $t=t_{f}$, for a rigid section if $0 \leq x \leq x_{1}\left(t_{f}\right)$

$$
w\left(x, t_{f}\right)=\left(f_{01}\left(x_{1}\left(t_{f}\right)\right)\left(f_{4} e^{f_{2} t_{f}}+f_{6} e^{f_{3} t_{f}}+f_{8} e^{-\alpha_{1} t_{f}}\right)+f_{5} e^{f_{2} t_{f}}+f_{7} e^{f_{3} t_{f}}+f_{9}\right) \frac{x}{x_{1}\left(t_{f}\right)},
$$


and plastic area if $x_{1}\left(t_{f}\right) \leq x \leq \frac{a}{2}$ from equation (25)

$$
w(x, t)=f_{01}(x)\left(f_{4} e^{f_{2} t_{f}}+f_{6} e^{f_{3} t_{f}}+f_{8} e^{-\alpha_{1} t_{f}}\right)+f_{5} e^{f_{2} t_{f}}+f_{7} e^{f_{3} t_{f}}+f_{9} .
$$

In the case of the second charge, at the time $t=t_{1}$ when the beam movement has not stopped $t_{1}<t_{f}$, the load caused by the action of two charges takes the form

$$
q(x, t)=f_{01}(x) e^{-\alpha_{1} t}+f_{02}(x) e^{-\alpha_{2} t},
$$

then the equation for determining the deflection in the plastic area takes the form

$$
\frac{\partial^{2} w}{\partial t^{2}}+\frac{k_{1}}{m} \frac{\partial w}{\partial t}+\frac{k_{2}}{m} w=f_{01}(x) e^{-\alpha_{1} t}+f_{02}(x) e^{-\alpha_{2} t}+f_{1},
$$

solution (33) has the form

$$
w(x, t)=C_{3}(x) e^{f_{2} t}+C_{4}(x) e^{f_{3} t}+\frac{f_{01}(x) e^{-\alpha_{1} t}}{\left(f_{2}+\alpha_{1}\right)\left(f_{3}+\alpha_{1}\right)}+\frac{f_{02}(x) e^{-\alpha_{2} t}}{\left(f_{2}+\alpha_{2}\right)\left(f_{3}+\alpha_{2}\right)}+\frac{f_{1}}{f_{2} f_{3}},
$$

under initial conditions

$$
\left.w(x, t)\right|_{t=t_{1}}=w_{01}(x),\left.\frac{\partial w(x, t)}{\partial t}\right|_{t=t_{1}}=w_{02}(x)
$$

where

$$
\begin{gathered}
w_{01}(x)=f_{10} f_{01}(x)+f_{11}, w_{02}(x)=f_{12} f_{01}(x)+f_{13}, \\
f_{10}=f_{4} e^{f_{2} t_{1}}+f_{6} e^{f_{3} t_{1}}+f_{8} e^{-\alpha_{1} t_{1}}, f_{11}=f_{5} e^{f_{2} t_{1}}+f_{7} e^{f_{3} t_{1}}+f_{9}, \\
f_{12}=f_{4} f_{2} e^{f_{2} t_{1}}+f_{6} f_{3} e^{f_{3} t_{1}}-\alpha_{1} f_{8} e^{-\alpha_{1} t_{1}}, f_{13}=f_{5} f_{2} e^{f_{2} t_{1}}+f_{7} f_{3} e^{f_{3} t_{1}},
\end{gathered}
$$

the solution takes the form

$$
\begin{aligned}
w(x, t)=\left(f_{14} f_{01}(x)\right. & \left.+f_{15} f_{02}(x)+f_{16}\right) e^{f_{2} t}+\left(f_{17} f_{01}(x)+f_{18} f_{02}(x)+f_{19}\right) e^{f_{3} t}+ \\
& +f_{20} e^{-\alpha_{1} t} f_{01}(x)+f_{21} e^{-\alpha_{2} t} f_{02}(x)+f_{22}
\end{aligned}
$$

where

$$
\begin{gathered}
f_{14}=\frac{e^{-\left(\alpha_{1}+f_{2}\right) t_{1}}}{\left(f_{2}+\alpha_{1}\right)\left(f_{2}-f_{3}\right)}+\frac{e^{-f_{2} t_{1}}\left(f_{12}-f_{3} f_{10}\right)}{f_{2}-f_{3}}, f_{15}=\frac{e^{-\left(\alpha_{2}+f_{2}\right) t_{1}}}{\left(f_{2}+\alpha_{2}\right)\left(f_{2}-f_{3}\right)}, \\
f_{16}=\frac{f_{1} e^{-f_{2} t_{1}}}{f_{2}\left(f_{2}-f_{3}\right)}+\frac{e^{-f_{2} t_{1}}\left(f_{13}-f_{3} f_{11}\right)}{f_{2}-f_{3}}, f_{17}=\frac{e^{-\left(\alpha_{1}+f_{3}\right) t_{1}}}{\left(f_{3}+\alpha_{1}\right)\left(f_{3}-f_{2}\right)}+\frac{e^{-f_{3} t_{1}}\left(f_{12}-f_{2} f_{10}\right)}{f_{3}-f_{2}}, \\
f_{18}=\frac{e^{-\left(\alpha_{2}+f_{3}\right) t_{1}}}{\left(f_{3}+\alpha_{2}\right)\left(f_{3}-f_{2}\right)}, f_{19}=\frac{f_{1} e^{-f_{3} t_{1}}}{f_{3}\left(f_{3}-f_{2}\right)}+\frac{e^{-f_{3} t_{1}}\left(f_{13}-f_{2} f_{11}\right)}{f_{3}-f_{2}} \\
f_{20}=\frac{1}{\left(f_{2}+\alpha_{1}\right)\left(f_{3}+\alpha_{1}\right)}, f_{21}=\frac{1}{\left(f_{2}+\alpha_{2}\right)\left(f_{3}+\alpha_{2}\right)}, f_{22}=\frac{f_{1}}{f_{2} f_{3}} .
\end{gathered}
$$

The obtained solution we rewrite as

$$
\begin{gathered}
w(x, t)=f_{01}(x)\left(f_{14} e^{f_{2} t}+f_{17} e^{f_{3} t}+f_{20} e^{-\alpha_{1} t}\right)+f_{02}(x)\left(f_{15} e^{f_{2} t}+f_{18} e^{f_{3} t}+f_{21} e^{-\alpha_{1} t}\right)+ \\
+f_{16} e^{f_{2} t}+f_{19} e^{f_{3} t}+f_{22} .
\end{gathered}
$$

From (17), (27), (35) it is possible to obtain a solution in a rigid part

$$
\begin{gathered}
w(x, t)=\left(f_{01}\left(x_{2}(t)\right)\left(f_{14} e^{f_{2} t}+f_{17} e^{f_{3} t}+f_{20} e^{-\alpha_{1} t}\right)+\right. \\
\left.+f_{02}\left(x_{2}(t)\right)\left(f_{15} e^{f_{2} t}+f_{18} e^{f_{3} t}+f_{21} e^{-\alpha_{1} t}\right)+\left(f_{16} e^{f_{2} t}+f_{19} e^{f_{3} t}+f_{22}\right)\right) \frac{x}{x_{2}(t)}
\end{gathered}
$$


The equation for determining the boundary between two regions can be obtained according to (29), (35), (36)

$$
\begin{gathered}
\left(f_{01}\left(x_{2}(t)\right)-\left.x_{2}(t) f_{01}^{\prime}(x)\right|_{x=x_{2}(t)}\right)\left(f_{14} e^{f_{2} t}+f_{17} e^{f_{3} t}+f_{20} e^{-\alpha_{1} t}\right)+ \\
+\left(f_{02}\left(x_{2}(t)\right)-\left.x_{2}(t) f_{02}^{\prime}(x)\right|_{x=x_{2}(t)}\right)\left(f_{15} e^{f_{2} t}+f_{18} e^{f_{3} t}+f_{21} e^{-\alpha_{1} t}\right)+\left(f_{16} e^{f_{2} t}+f_{19} e^{f_{3} t}+f_{22}\right)=0 .
\end{gathered}
$$

The stopping time of structure motion $t_{f_{2}}$ after the second explosion can be determined from the equation

$$
\begin{gathered}
f_{01}\left(x_{2}\left(t_{f_{2}}\right)\right)\left(f_{14} f_{2} e^{f_{2} t_{f_{2}}}+f_{17} f_{3} e^{f_{3} t_{f_{2}}}-\alpha_{1} f_{20} e^{-\alpha_{1} t_{f_{2}}}\right)+ \\
+f_{02}\left(x_{2}\left(t_{f_{2}}\right)\right)\left(f_{15} f_{2} e^{f_{2} t_{f_{2}}}+f_{18} f_{3} e^{f_{3} t_{2}}-\alpha_{1} f_{21} e^{-\alpha_{1} t_{2}}\right)+f_{16} f_{2} e^{f_{2} t_{f_{2}}}+f_{19} f_{3} e^{f_{3} t_{f_{2}}}=0 .
\end{gathered}
$$

The residual deflection of structure after motion stop will take the form: for $0 \leq x \leq x_{2}\left(t_{f}\right)$

$$
\begin{gathered}
w_{f 2}(x)=f_{01}(x)\left(f_{14} e^{f_{2} t_{f_{2}}}+f_{17} e^{f_{3} t_{f_{2}}}+f_{20} e^{-\alpha_{1} t_{f_{2}}}\right)+f_{02}(x)\left(f_{15} e^{f_{2} t_{f_{2}}}+f_{18} e^{f_{3} t_{f_{2}}}+f_{21} e^{-\alpha_{1} t_{f_{2}}}\right)+ \\
+f_{16} e^{f_{2} t_{f_{2}}}+f_{19} e^{f_{3} t_{f_{2}}}+f_{22},
\end{gathered}
$$

for $x_{2}\left(t_{f}\right) \leq x \leq \frac{a}{2}$

$$
\begin{gathered}
w_{f 2}(x)=\left(f_{01}\left(x_{2}\left(t_{f_{2}}\right)\right)\left(f_{14} e^{f_{2} t_{f_{2}}}+f_{17} e^{f_{3} t_{f_{2}}}+f_{20} e^{-\alpha_{1} t_{f_{2}}}\right)+\right. \\
\left.+f_{02}\left(x_{2}\left(t_{f_{2}}\right)\right)\left(f_{15} e^{f_{2} t_{f_{2}}}+f_{18} e^{f_{3} t_{f_{2}}}+f_{21} e^{-\alpha_{1} t_{f_{2}}}\right)+\left(f_{16} e^{f_{2} t_{f_{2}}}+f_{19} e^{f_{3} t_{f_{2}}}+f_{22}\right)\right) \frac{x}{x_{2}\left(t_{f_{2}}\right)} .
\end{gathered}
$$

The value of the bending moment in the rigid part of the beam can be determined from the equations (17), (18), (28), (36). The moment should not exceed the limit value $M(x, t)<M_{0}^{+}$in this part .

\section{Discussion}

The approach used in this work allows to consider sections consisting of three layers as well as more. An increase of the number of layers does not introduce any serious complications in the calculation and does not change the algorithm for solving the problem. The considered approach can also be used in the case when each concrete layer is reinforced with high-modulus and high-strength fibers of metal, polymer or stone origin (at creating poly-reinforced structures), as well as using rubber-concrete and polymer concrete.

\section{Conclusions}

The problem of bending of a multilayered concrete beam of arbitrary cross section by explosive load on a visco-elastic basis has been set and solved under the assumption that in the section different grades of concrete can be realized in layers. The distribution of bending moments and deflections of the beam at each time is determined. The time of the end of motion and the residual deflection of beam are found.

This work is carried out with the partial financial support of RFBR grants (projects 19-01-00038, 1741-210272). 


\section{References}

1. A. A. Gvozdev, Building industry, 1, 18-21 (1940)

2. M. I. Erkhov, Theory of perfectly plastic solids and structures (Nauka, Moskow, 1978)

3. V. M. Shamin, Calculation of elements of constructions on action of explosive loadings (Stroyizdat, Moskow, 1989)

4. K. L. Komarov, Yu.V. Nemirovskii, Dynamics of rigid and plastic elements of designs, (Nauka, Novosibirsk, 1984)

5. N. Johns, T. O. Uran, S. A. Tekin, Int. J. Solid Struct., 12, 1499-1512 (1970)

6. A. D. Cox, W. D. Morland, Journ. Mech. Solids, 7, 229-241 (1959)

7. Y. V. Nemirovskii, A. I. Boltaev, Bulletin of BSTU named after V.G. Shukhov, 6, 125129 (2016)

8. Y. V. Nemirovskii, A. I. Boltaev, Wschodnioeuropejskie czasopismo naukowe, 3, 151161 (2016)

9. Y. V. Nemirovskii, S. V. Tikhonov, Bulletin of the Yakovlev Chuvash State Pedagogical University. Series: Mechanics of Limit State, 3, 134-158 (2016)

10. M. I. Gorbunov-Posadov Beams and plates on the elastic foundation (Mashstroyizdat, Moscow, 1949)

11. Y. V. Nemirovskii, S. V. Tikhonov, Bulletin of the Yakovlev Chuvash State Pedagogical University. Series: Mechanics of Limit State, 2, 45-64 (2017)

12. Y. V. Nemirovsky, S. V. Tikhonov, Journal of Physics: Conference Series, 973, 012014 (2018)

13. Y. V. Nemirovskii, Bulletin of the Yakovlev Chuvash State Pedagogical University. Series: Mechanics of Limit State, 3, 26-37 (2018)

14. Y. V. Nemirovskii, Bulletin of the Yakovlev Chuvash State Pedagogical University. Series: Mechanics of Limit State, 2, 78-84 (2014)

15. A. V. Pinaev, V. T. Kuzavov, V. K. Kedrinsky, Journal of Applied Mechanics and Technical Physics, 5, 81-90, (2000) 